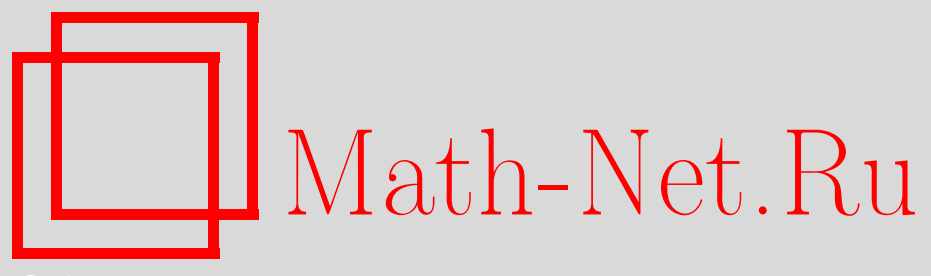

Х. Ф. Кариньена, Х. Клементе-Гайардо, Д. Мармо, Геометризация квантовой механики, ТМФ, 2007, том 152, номер 1, 20-31

DOI: https://doi.org/10.4213/tmf6067

Использование Общероссийского математического портала Math-Net.Ru подразумевает, что вы прочитали и согласны с пользовательским соглашением http://www . mathnet.ru/rus/agreement

Параметры загрузки:

IP : 3.91 .87 .62

26 апреля 2023 г., 16:21:14

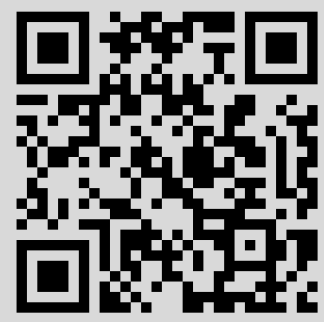




\title{
ГЕОМЕТРИЗАЦИЯ КВАНТОВОЙ МЕХАНИКИ
}

\begin{abstract}
Показано, что различные описания квантовой механики можно представить в геометрических терминах. В частности, на пространстве наблюдаемых с использованием отображения импульсов, связанного с унитарной группой, получено унифицированное геометрическое описание для различных картин квантовой механики. Такая конструкция предлагает альтернативу обычной конструкции Гельфанда-Наймарка-Сегала для чистых состояний.
\end{abstract}

Ключевые слова: квантовая механика, эрмитова структура, алгебра Йордана, скобка Пуассона, алгебра Ли-Йордана.

\section{1. ВВЕДЕНИЕ}

Описание любой физической системы, классической или квантовой, требует указания пространства состояний $\mathcal{S}$, пространства наблюдаемых $\mathcal{O}$ и их спаривания, результатом которого является число в поле вещественных чисел $\mathbb{R}$. Такое спаривание можно интерпретировать как процесс измерения.

Обычно в классической механике пространство состояний наделено структурой вещественного дифференцируемого многообразия, пространство наблюдаемых отождествляется с пространством функций на многообразии, а спаривание задается применением функции к состоянию. Альтернативные подходы исходят из ассоциативной коммутативной алгебры функций (наблюдаемых), причем состояния отождествляются с гомоморфизмами из алгебры наблюдаемых в алгебру вещественных чисел. За такой подход выступал в нескольких статьях Виноградов; сообщение об этом можно найти в работе [1].

Согласно Шредингеру и Дираку для квантовых систем исходным объектом является пространство состояний $\mathcal{S}$. Оно обычно отождествляется с сепарабельным

*Departamento de Física Teórica, Universidad de Zaragoza, 50009 Zaragoza, Spain. E-mail: jfc@unizar.es

${ }^{\dagger}$ Instituto de Biocomputación y Física de los Sistemas complejos, Universidad de Zaragoza, 50009 Zaragoza, Spain. E-mail: jcg@unizar.es

${ }^{\ddagger}$ Dipartimento di Scienze Fisiche, Università Federico II di Napoli; INFN, Sezione di Napoli, Complesso Universitario di Monte Sant'Angelo, Via Cintia, 80126 Napoli, Italy.

E-mail:marmo@na.infn.it 
комплексным гильбертовым пространством $\mathcal{H}$ [2]. Наблюдаемые являются вторичным объектом и отождествляются с самосопряженными операторами, действующими на $\mathcal{H}$ (проблема с областью определения сейчас для нас не важна). Спаривание задается ожидаемыми значениями наблюдаемых на элементах пространства $\mathcal{H}$.

Отправной точкой альтернативного подхода, представляющего собой развитие гейзенберговского описания квантовой механики в терминах "бесконечномерных матриц" согласно, в основном, работам Сегала [3], Хаага и Кастлера [4] является $\mathbb{C}^{*}$-алгебра, а наблюдаемые (вещественные динамические переменные) даются ее самосопряженными элементами. Состояния при этом строятся как линейные функционалы на алгебре, подчиненные дополнительным требованиям, для того чтобы их применение к наблюдаемым можно было интерпретировать как измерительную процедуру.

Итак, представляется, что в то время как классическая механика в своих формулировках обладает существенным геометрическим содержанием, квантовую механику приходится формулировать в основном в терминах (линейных) алгебраических структур. Группа преобразований классической механики является подгруппой группы диффеоморфизмов многообразия состояний (в духе эрлангенской программы Клейна), при этом соответствующая группа квантовой механики является подгруппой линейных преобразований. Однако общепринятым является мнение о том, что классическая механика должна быть подходящим пределом квантовой механики (обычно это известно как “принцип соответствия"). Поэтому разумно ожидать, что лучшего понимания этой "предельной процедуры" можно достичь, если разработать "геометризацию" квантовой механики так, чтобы можно было выйти за рамки группы линейных преобразований. Ранее были предприняты попытки геометрической формулировки квантовой механики [5]-[15], однако авторы этих работ подходили к решению проблемы с другой точки зрения: они исходили из гильбертова пространства состояний и пытались отождествить необходимые геометрические структуры, возникающие из эрмитовой структуры.

Цель настоящей работы состоит в том, чтобы наметить еще один путь достижения геометризации. Мы будем следовать близкому к упомянутому выше $\mathbb{C}^{*}$-алгебраическому подходу. Чтобы избежать технических подробностей, которые могут скрыть вводимые нами математические геометрические структуры, мы ограничимся конечноуровневыми квантовыми системами. Мы надеемся, что в этом случае геометрические структуры будут более четкими.

Нашим исходным объектом будет семейство эрмитовых операторов (наблюдаемых) в пространстве $\mathcal{H}$, находящееся во взаимно однозначном соответствии с вещественной алгеброй Ли $\mathfrak{u}(\mathcal{H})$ унитарной группы $U(\mathcal{H})$. Вещественное векторное пространство $\mathfrak{u}(\mathcal{H})$ обладает структурой не только алгебры Ли, но и алгебры Йордана [16], причем эти две структуры совместны в смысле, который будет ясен ниже.

"Геометризация" структуры алгебры Ли на пространстве наблюдаемых легко достигается путем замены коммутатора Ли на линейный тензор Пуассона, определенный на двойственном пространстве $\mathfrak{u}^{*}(\mathcal{H})$ алгебры Ли $\mathfrak{u}(\mathcal{H})$. Аналогичным образом мы также геометризуем структуру алгебры Йордана, определив соответствующий тензор на том же двойственном векторном пространстве. При такой замене мы 
больше не ограничены одними только линейными преобразованиями, однако $\mathfrak{u}(\mathcal{H})$ и $\mathfrak{u}^{*}(\mathcal{H})$ рассматриваются как $n^{2}$-мерные многообразия.

Используя заданную структуру пуассонова многообразия на таком пространстве, мы можем искать ее симплектические реализации. В частности, симплектическая реализация с помощью симплектического векторного пространства заменяет, исходя из чистого состояния, более знакомую конструкцию Гельфанда-Наймарка-Сегала (ГНС) гильбертова пространства, связанную с $\mathbb{C}^{*}$-алгеброй динамических переменных.

Симплектическое векторное пространство, снабженное подходящей комплексной структурой, порождает гильбертово пространство $\mathcal{H}$. Симплектическое действие соответствующей унитарной группы дает отображение импульсов $\mu: \mathcal{H} \rightarrow \mathfrak{u}^{*}(\mathcal{H})$, которое определяет искомую симплектическую реализацию пространства наблюдаемых. Мы свяжем пространство чистых состояний с комплексным проективным пространством, которое оказывается симплектоморфным минимальным симплектическим орбитам соответствующего коприсоединенного действия (унитарной группы) на пространстве $\mathfrak{u}^{*}(\mathcal{H})$.

\section{2. ПРОСТРАНСТВО НАБЛЮДАЕМЫХ}

Следуя алгебраическому подходу, предложенному Сегалом [3], а также Хаагом и Кастлером [4], рассмотрим множество наблюдаемых как набор всех самосопряженных элементов $\mathbb{C}^{*}$-алгебры с единицей. Тогда множество состояний $\mathcal{S}$ является набором всех положительных вещественных значений линейных функционалов $\Phi$, нормированных условием

$$
\Phi(\mathbb{I})=1 .
$$

Спаривание этих двух множеств определяется вычислением состояния, т.е. взятием ожидаемого значения наблюдаемой, когда состоянием системы является $\Phi$.

2.1. Алгебраические структуры. Имеется следующий хорошо известный результат: каждую $\mathbb{C}^{*}$-алгебру с единицей можно реализовать как множество всех ограниченных операторов, действующих на некотором гильбертовом пространстве $\mathcal{H}$ (детали см. в работе [17]). Поскольку мы решили работать с конечноуровневыми квантовыми системами, отождествим множество наблюдаемых с пространством эрмитовых операторов (т.е. операторов, удовлетворяющих условию $A^{\dagger}=A$, которое теперь следует записать как $A^{*}=A$ для $\mathbb{C}^{*}$-инволюции). Они определяют вещественное векторное пространство, изоморфное алгебре $\mathfrak{u}(\mathcal{H})$. Эта алгебра соответствует антиэрмитовым операторам, которые в случае конечномерных систем определяют алгебру Ли $\mathfrak{u}(n)$, где $n$ - размерность гильбертова пространства.

Умножение на мнимую единицу $i$ устанавливает изоморфизм множества наблюдаемых (как векторного пространства) и вещественного векторного пространства алгебры Ли унитарной группы $\mathfrak{u}(\mathcal{H})$ :

$$
\alpha: \mathcal{O} \rightarrow \mathfrak{u}(\mathcal{H}) \quad \alpha(A)=i A .
$$

В результате, поскольку мы рассматриваем случай конечномерного гильбертова пространства, можно немедленно доказать, что пространство $\mathcal{O}$ можно отождествить с пространством $\mathfrak{u}^{*}(\mathcal{H})$. Суммируем этот результат в виде леммы. 
ЛЕмма 1. Пространство наблюдаемых $\mathcal{O}$ наделяется структурой алгебры Ли, изоморфной естественной структуре на пространстве $\mathfrak{u}(\mathcal{H})$, если положить

$$
[A, B]_{-}=\alpha^{-1}(\alpha(A) \alpha(B)-\alpha(B) \alpha(A))=-i[\alpha(A), \alpha(B)]
$$

и задать вещественное скалярное произведение, инвариантное относительно присоединенного представления, как

$$
\langle A, B\rangle=\frac{1}{2} \operatorname{Tr}(A B)
$$

Еще один изоморфизм, отождествляющий векторные пространства $\mathfrak{u}(\mathcal{H})$ и $\mathfrak{u}^{*}(\mathcal{H})$, можно определить в соответствии со спариванием, задаваемым формой КиллингаКартана

$$
\xi(A)=\frac{i}{2} \operatorname{Tr}(\xi A), \quad \xi \in \mathfrak{u}^{*}(\mathcal{H}), \quad A \in \mathfrak{u}(\mathcal{H}) .
$$

Пространство эрмитовых операторов содержит также другое бинарное произведение (обычно называемое йордановым произведением), которое определяется как

$$
A \circ B=\frac{1}{2}(A B+B A)=[A, B]_{+} .
$$

Напомним для полноты определение алгебры Йордана.

ОПРЕДЕЛЕНиЕ 1. Неассоциативная алгебра $(\mathcal{A}, \cdot)$ называется алгеброй Йордана, если операция является коммутативной, и для двух произвольных элементов $x, y \in$ $\mathcal{A}$ выполнено $(x y) x^{2}=x\left(y x^{2}\right)$.

На основании такого определения можно вывести следующую лемму.

Лемма 2. Алгебра $(\mathcal{O}$, о) является алгеброй Йордана.

ДокАЗАТЕЛьство. Коммутативность доказывается тривиально. Второе условие также довольно просто получить. В самом деле, рассмотрим $x, y \in \mathcal{O}$, тогда

$$
\begin{aligned}
(x \circ y) \circ x^{2} & =\left[[x, y]_{+}, x^{2}\right]_{+}=2(x y+y x) x^{2}+2 x^{2}(x y+y x)= \\
& =2\left(x y x^{2}+y x^{3}+x^{3} y+x^{2} y x\right), \\
x \circ\left(y \circ x^{2}\right) & =\left[x,\left[y, x^{2}\right]_{+}\right]_{+}=2 x\left(y x^{2}+x^{2} y\right)+\left(y x^{2}+x^{2} y\right) x= \\
& =2\left(x y x^{2}+x^{3} y+y x^{3}+x^{2} y x\right) .
\end{aligned}
$$

ПРЕДЛОЖЕНИЕ 1. СКалярное произведение (4) инвариантно относительно произведения (6), и мы имеем

$$
\left\langle[A, B]_{-}, C\right\rangle=\left\langle A,[B, C]_{-}\right\rangle, \quad\left\langle[A, B]_{+}, C\right\rangle=\left\langle A,[B, C]_{+}\right\rangle .
$$

Более того, имеется соотношение совместности

$$
[A, B \circ C]_{-}=[A, B]_{-} \circ C+B \circ[A, C]_{-},
$$

m.е. $\mathrm{ad}_{-}$является дифференцированием алгебры Йордана. 
ДоКАЗАТЕЛЬСТво этих свойств следует непосредственно из определений.

В соответствии с работами [17], [18] введем следующее определение.

ОПредЕЛЕНиЕ 2. Алгебра Ли $\left(\mathcal{A},[\cdot, \cdot]_{-}\right)$называется алгеброй Ли-Йордана, если она допускает структуру алгебры Йордана, удовлетворяющую (8) и тождеству ассоциативности при некотором $\hbar \in \mathbb{R}:$

$$
(A \circ B) \circ C-A \circ(B \circ C)=\frac{1}{4} \hbar\left[[A, C]_{-}, B\right]_{-} \quad \forall A, B, C \in \mathcal{A} .
$$

ЛЕмма 3 [17]. Пространство наблюдаемых с определенными выше операчиями также становится алгеброй Ли-Йордана.

2.2. Геометрические структуры. Конкретный способ геометризации структуры алгебры Ли на пространстве $\mathfrak{u}(\mathcal{H})$ состоит в том, чтобы следующим образом связать с ней линейный тензор Пуассона на двойственном векторном пространстве $\mathfrak{u}^{*}(\mathcal{H})$. Поскольку $\mathcal{H}$ предполагается конечномерным, можно отождествить $\mathfrak{u}(\mathcal{H})$ с пространством вещественнозначных линейных функций на его двойственном пространстве, т.е. $\mathfrak{u}(\mathcal{H}) \sim \operatorname{Lin}\left(\mathfrak{u}^{*}(\mathcal{H}), \mathbb{R}\right)$, и для любой пары линейных функций на $\mathfrak{u}^{*}(\mathcal{H})$, определенных двумя элементами $u, v \in \mathfrak{u}(\mathcal{H})$,

$$
\{\hat{u}, \hat{v}\}=\widehat{[u, v]}
$$

где при вычислении коммутатора надо считать $u$ и $v$ в правой части элементами алгебры Ли $\mathfrak{u}(\mathcal{H})$, а в левой части линейными функциями на $\mathfrak{u}^{*}(\mathcal{H})$. Мы будем использовать шляпку для обозначения элементов пространства $\mathfrak{u}(\mathcal{H})$, рассматриваемых как линейные функции на двойственном пространстве $\mathfrak{u}^{*}(\mathcal{H})$. Здесь мы неявно используем свойство, что векторное пространство $\mathfrak{u}(\mathcal{H})$ изоморфно своему дважды двойственному; это свойство выполнено для рефлексивных векторных пространств, в частности, конечномерных. Таким образом, мы имеем

ПРЕДЛОЖЕНИЕ 2. Пусть $\mathcal{O}$ - пространство наблюдаемых квантовой системы конечного уровня. Тогда $\mathcal{O}^{*}($ и само $\mathcal{O})$ можно наделить пуассоновой структурой.

После замены структуры алгебры Ли тензором Пуассона, связанным со скобками Пуассона на $\mathfrak{u}^{*}(\mathcal{H})$, можно выполнять нелинейные преобразования на пуассоновом многообразии. В этом смысле мы говорим о "геометризации" алгебраической структуры векторного пространства $\mathfrak{u}(\mathcal{H})$.

Если обозначить через $\hat{A}$ и $\widehat{B}$ линейные функции на пространстве $\mathfrak{u}^{*}(\mathcal{H})$, соответствующие элементам $A, B \in \mathfrak{u}(\mathcal{H})$, можно определить пуассонов бивектор $\Lambda$ :

$$
\Lambda(d \hat{A}, d \widehat{B})(\xi)=\{\hat{A}, \widehat{B}\}(\xi)=\xi([A, B])=\frac{i}{2} \operatorname{Tr} \xi(A B-B A), \quad \xi \in \mathfrak{u}^{*}(\mathcal{H}),
$$

где мы использовали соотношение (5). Таким образом воспроизводится хорошо известный пуассонов тензор Кириллова-Константа-Сурьо на пространстве, двойственном ко всякой алгебре Ли. 
Используя аналогичную процедуру, можно также “геометризовать" структуру алгебры Йордана на пространстве наблюдаемых. Снова положим

$$
\mathcal{R}(\xi)(d \hat{A}, d \widehat{B})=\xi\left([A, B]_{+}\right)=\frac{1}{2} \operatorname{Tr} \xi(A B+B A), \quad \xi \in \mathfrak{u}^{*}(\mathcal{H}),
$$

где мы использовали соотношение (5). Два тензорных поля (10) и (11) можно объединить в комплексное векторное поле

$$
(\mathcal{R}+i \Lambda)(\xi)(d \hat{A}, d \widehat{B})=2(\widehat{A B})(\xi)=\xi(A B)=\operatorname{Tr}(\xi A B), \quad \xi \in \mathfrak{u}^{*}(\mathcal{H}) .
$$

Используя это тензорное поле, можно определить $*$-произведение как

$$
(\hat{A} * \widehat{B})(\xi)=\xi(A B)=(\mathcal{R}+i \Lambda)(\xi)(d \hat{A}, d \widehat{B}) .
$$

В данном контексте условие совместности структур алгебр Ли и Йордана можно установить следующим простым способом.

ПреДЛОЖЕНИЕ 3. Гамильтоновы векторные поля, связанные с наблюдаемыми (т.е. линейнъе функции на пространстве $\mathfrak{u}^{*}(\mathcal{H})$ ), являются инфинитезимальнъми симметриями тензорного поля $\mathcal{R}$, связанного со структурой алгебры Йордана, а потому дифференцируют *-произведение.

ДокАЗАтЕЛЬСтво. Это утверждение является прямым следствием совместности скобок, см. (8).

2.3. Распределения. В этом пункте мы кратко суммируем результаты работ [19], [20], касающиеся подходящих распределений, определенных на $\mathfrak{u}(\mathcal{H})$, которые соответствуют рассмотренным выше геометрическим объектам. Если использовать отождествление вещественного векторного пространства $\mathfrak{u}(\mathcal{H})$ с его касательным пространством в любой точке, можно определить $(1,1)$-тензорное поле $\hat{J}$ в $\mathfrak{u}(\mathcal{H})$, полагая

$$
\hat{J}_{\xi}(A)=[A, \xi]_{-}=\Lambda_{\xi}(d \hat{A}), \quad A \in \mathfrak{u}(\mathcal{H})
$$

Аналогично можно записать

$$
\widehat{\mathcal{R}}_{\xi}(A)=[A, \xi]_{+}=\mathcal{R}_{\xi}(d \hat{A}), \quad A \in \mathfrak{u}(\mathcal{H}) .
$$

Эти два тензора позволяют определить два важных распределения:

$$
D_{\Lambda}=(\hat{J}), \quad D_{\mathcal{R}}=(\widehat{\mathcal{R}}) .
$$

Рассматривая эти два объекта, находим, что оба тензора коммутируют между собой:

$$
\hat{J} \circ \widehat{\mathcal{R}}(A)=\widehat{\mathcal{R}} \circ \hat{J}(A)=\frac{1}{2}\left[A, \xi^{2}\right]_{-} .
$$

Из двух распределений можно скомбинировать два новых: $D_{0}=D_{\mathcal{R}} \cap D_{\Lambda}$ и $D_{1}=D_{\mathcal{R}}+D_{\Lambda}$.

ПреДлОЖение 4 [19]. Распределения $D_{0}, D_{1}, D_{\mathcal{R}}$ u $D_{\Lambda}$ удовлетворяют следующим условиям: 
- Распределение $D_{\Lambda}$ является инволютивным и порождает обобщенное слоение, листы которого являются симплектическими многообразиями, связанными с пуассоновым тензором $\Lambda$.

- Распределение $D_{\mathcal{R}}$ не является инволютивным.

- Распределение $D_{0}$ является инволютивным. Соответствующее слоение инвариантно относительно присоединенного представления, а каждый лист является кэлеровым многообразием.

- Распределение $D_{1}$ является инволютивным, а соответствующие листы связанного с ним слоения представляют собой орбиты $G L(\mathcal{H})$-действия, которое определяется как

$$
G L(\mathcal{H}) \times \mathfrak{u}(\mathcal{H}) \rightarrow \mathfrak{u}(\mathcal{H}), \quad(T, A) \mapsto T A T^{\dagger} .
$$

ЗАмечАниЕ 1. Наша "геометризация" дает возможность выполнять нелинейные преобразования, поскольку мы заменили алгебраические структуры на линейном пространстве $\mathfrak{u}(\mathcal{H})$ тензорными объектами на многообразии $u^{*}(\mathcal{H})$. Однако следует заметить, что для линейных функций на $\mathfrak{u}^{*}(\mathcal{H})$ теперь могут быть определены два различных произведения: поточечное произведение $(\hat{A} \cdot \widehat{B})(\xi)=\hat{A}(\xi) \widehat{B}(\xi)$, которое дает квадратичную функцию на основании двух линейных, и нелокальное произведение $(\hat{A} \star \widehat{B})(\xi)=\widehat{A B}(\xi)$. В последнем случае произведение линейных функций дает линейную, но, вообще говоря, комплекснозначную функцию, даже если множители будут вещественными; это связано с тем, что произведение двух эрмитовых операторов не является эрмитовым оператором, поэтому возникают вещественная и мнимая части.

ПримеР. Рассмотрим алгебру Ли $\mathfrak{s u}(2)$ эрмитовых $(2 \times 2)$-матриц, соответствующих физической системе со спином $1 / 2$. Введем ортонормированный базис относительно скалярного произведения (4). Таким образом, мы полагаем

$$
U=\left(\begin{array}{ll}
1 & 0 \\
0 & 1
\end{array}\right), \quad X=\left(\begin{array}{cc}
0 & 1 \\
1 & 0
\end{array}\right), \quad Y=\left(\begin{array}{cc}
0 & -i \\
i & 0
\end{array}\right), \quad Z=\left(\begin{array}{cc}
1 & 0 \\
0 & -1
\end{array}\right),
$$

при этом соответствующие линейные функции имеют вид

$$
\widehat{X}=x, \quad \widehat{Y}=y, \quad \widehat{Z}=z, \quad \widehat{U}=u,
$$

где функции понимаются как $z(A)=(i / 2) \operatorname{Tr}(Z A)$ для всех $A \in u(2)$. В этих координатах пуассоново тензорное поле имеет вид

$$
\Lambda=2\left(x \frac{\partial}{\partial y} \wedge \frac{\partial}{\partial z}+y \frac{\partial}{\partial z} \wedge \frac{\partial}{\partial x}+z \frac{\partial}{\partial x} \wedge \frac{\partial}{\partial y}\right)
$$

а тензор йордановой структуры задается как

$$
\begin{aligned}
\mathcal{R}=2 & \frac{\partial}{\partial u} \otimes_{\mathrm{s}}\left(x \frac{\partial}{\partial x}+y \frac{\partial}{\partial y}+z \frac{\partial}{\partial z}\right)+ \\
& +2 u\left(\frac{\partial}{\partial u} \otimes_{\mathrm{s}} \frac{\partial}{\partial u}+\frac{\partial}{\partial x} \otimes_{\mathrm{s}} \frac{\partial}{\partial x}+\frac{\partial}{\partial y} \otimes_{\mathrm{s}} \frac{\partial}{\partial y}+\frac{\partial}{\partial z} \otimes_{\mathrm{s}} \frac{\partial}{\partial z}\right)
\end{aligned}
$$


где $\otimes_{\mathrm{s}}$ означает симметризованное тензорное произведение. Сразу видно, что тензор $\mathcal{R}$ остается инвариантным под действием векторных полей вращения, задаваемых линейными гамильтоновыми функциями по отношению к пуассонову тензору $\Lambda$. Можно даже рассмотреть, например, нелокальное произведение, тогда получаем

$$
\widehat{Z} \star \widehat{Y}=-i \widehat{X}, \quad \widehat{X} \star \widehat{Y}=i \widehat{Z}, \quad \widehat{Z} \star \widehat{X}=i \widehat{Y} .
$$

Также легко видеть, что гамильтоновы векторные поля, связанные с линейными функциями, дают дифференцирования как точечного, так и нелокального произведений. Таким образом, соответствующие уравнения движения сами по себе не отличают квантовый случай от классического, и именно произведение различает коммутативную или некоммутативную природу пространства, а также локальность или нелокальность операции. Поэтому оно отличает и классическую механику от квантовой.

2.4. Динамика. Теперь можно записать уравнение движения в фазовом пространстве наблюдаемых. В случае чисто гейзенберговой картины оно записывается как

$$
\frac{d}{d t} A=\frac{1}{\hbar}[H, A]_{-} .
$$

Используя "геометризацию", т.е. действуя в терминах двойственного пространства $\mathfrak{u}^{*}(\mathcal{H})$, получаем

$$
\frac{d}{d t} \hat{A}=\frac{1}{\hbar}\{\widehat{H}, \hat{A}\}
$$

Поскольку на $\mathfrak{u}^{*}(\mathcal{H})$ имеются различные алгебраические структуры, важно исследовать совместность с ними дифференциальных уравнений.

ЛЕмма 4. Линейные дифференциальные уравнения, сохраняющие оба произведения, соответствуют инфинитезимальным генераторам унитарных преобразований.

2.5. Состояния. Состояния отождествляются с элементами выпуклого тела $\mathcal{S}=$ $\left\{\phi \in \mathcal{A}^{*} \mid \phi\left(A^{*} A\right) \geqslant 0, \forall A \in \mathcal{A} ; \phi(\mathbb{I})=1\right\}$. Это несложно показать в конечномерном случае, а для бесконечномерного случая имеется теорема Глисона, согласно которой любое состояние можно записать в виде $\phi(A)=\operatorname{Tr} \rho_{\phi} A$.

Более того, $\rho_{\phi}$ представляет собой неотрицательно определенный оператор из $\mathfrak{g l}(\mathcal{H})$, т.е. $\rho_{\phi} \in \mathfrak{g l}(\mathcal{H})$ можно записать в виде $\rho_{\phi}=T^{\dagger} T$ для некоторого $T \in \mathfrak{g l}(\mathcal{H})$, причем выполняется соотношение $\operatorname{Tr} \rho_{\phi}=1$. Таким образом, $\mathcal{S}$ является выпуклым телом в аффинной гиперплоскости в $\mathfrak{u}^{*}(\mathcal{H})$, определяемой уравнением $\operatorname{Tr} \rho_{\phi}=1$. Поэтому пространство, касательное к этой аффинной гиперплоскости в точке, отождествляется с пространством бесследовых эрмитовых операторов и находится во взаимно однозначном соответствии с алгеброй Ли группы $S U(\mathcal{H})$.

\section{3. СИМПЛЕКТИЧЕСКИЕ РЕАЛИЗАЦИИ ПУАССОНОВА МНОГООБРАЗИЯ $\mathfrak{u}^{*}$}

3.1. Представление в конечномерном случае. В общем случае для реконструкции представления в гильбертовом пространстве на основе "алгебраического 
описания" квантовых систем наряду с “геометризацией” можно использовать и ГНСконструкцию. Это требует введения состояния (функционала на алгебре наблюдаемых, удовлетворяющего некоторым условиям) и определения приспособленного для этой цели гильбертова пространства, в котором может быть представлена алгебра наблюдаемых.

В конечномерной ситуации имеется более простая альтернатива. Для построения представления в гильбертовом пространстве можно использовать приемы из дифференциальной геометрии гамильтоновых систем. Конкретно, мы ищем симплектическую реализацию пуассонова многообразия с помощью симплектического векторного пространства (классическое отображение Йордана-Швингера).

ОпРЕДЕЛЕниЕ 3. Симплектическая реализация пуассонова многообразия $(N,\{\cdot, \cdot\})$ является пуассоновым отображением $\Phi: M \rightarrow N$, где $(M, \omega)$ - симплектическое многообразие. Если $M$ - симплектическое векторное пространство, то мы имеем специальный случай, при этом $\Phi$ называется классическим отображением Йордана-Швингера [21].

3.1.1. Кэлерово пространство. Первый шаг состоит в рассмотрении размерности алгебры наблюдаемых. При заданной размерности $\mathfrak{u}(\mathcal{H})$, скажем, $n^{2}$, можно рассмотреть действие унитарной группы $U(n)$ на векторном пространстве $\mathbb{R}^{2 n}$, наделенном кэлеровой структурой - тройкой $(\omega, g, J)$. Здесь $\omega$ - точная симплектическая структура, $g$ - евклидова структура, а $J$ - ассоциированная комплексная структура. Рассмотрим то, что известно как "определяющее представление" группы $U(n)$.

Симплектическая структура допускает 1-форму потенциала, которая инвариантна относительно действия; тогда соответствующее отображение импульсов дает искомую симплектическую реализацию (см. [22]). Отображение импульсов $\mu: \mathbb{R}^{2 n} \rightarrow$ $\mathfrak{u}^{*}(n)$ эквивариантно относительно фундаментального действия группы $U(n)$ на $\mathbb{C}^{n} \approx \mathbb{R}^{2 n}$ и коприсоединенного действия на $\mathfrak{u}^{*}(n)$.

Если для $\mathbb{C}^{n}$ выбрать ортономированный базис $\left\{e_{1}, \ldots, e_{n}\right\}$, то координаты можно определить, полагая $\left\langle e_{k} \mid \psi\right\rangle=z_{k}(\psi)=\left(q_{k}+i p_{k}\right)(\psi)$, где мы использовали дираковские обозначения для бра- и кет-векторов.

3.1.2. Геометрические структуры. Можно видеть [19], что в этих координатах мы имеем контравариантную версию евклидовой структуры, задаваемую соотношением $G=\sum_{k=1}^{n}\left(\frac{\partial}{\partial q_{k}} \otimes \frac{\partial}{\partial q_{k}}+\frac{\partial}{\partial p_{k}} \otimes \frac{\partial}{\partial p_{k}}\right)$ с пуассоновым тензором $\Omega=\sum_{k=1}^{n}\left(\frac{\partial}{\partial q_{k}} \wedge \frac{\partial}{\partial p_{k}}\right)$, а комплексная структура есть $J=\sum_{k=1}^{n}\left(\frac{\partial}{\partial p_{k}} \otimes d q_{k}+\frac{\partial}{\partial q_{k}} \otimes d p_{k}\right)$. В терминах комплексных координат эрмитова структура имеет вид $h=\sum_{k=1}^{n} d \bar{z}_{k} \otimes d z_{k}$. Соответствующая контравариантная форма задается как

$$
G+i \Omega=\sum_{k=1}^{n}\left(\frac{\partial}{\partial q_{k}}-i \frac{\partial}{\partial q_{k}}\right) \otimes\left(\frac{\partial}{\partial q_{k}}+i \frac{\partial}{\partial q_{k}}\right)=4 \sum_{k=1}^{n} \frac{\partial}{\partial z_{k}} \otimes \frac{\partial}{\partial \bar{z}_{k}}
$$

Можно определить бинарные произведения на функциях, полагая

$$
\left\{f_{1}, f_{2}\right\}=\sum_{k=1}^{n}\left(\frac{\partial f_{1}}{\partial q_{k}} \frac{\partial f_{2}}{\partial p_{k}}-\frac{\partial f_{1}}{\partial p_{k}} \frac{\partial f_{2}}{\partial q_{k}}\right)
$$




$$
\begin{aligned}
\left\{f_{1}, f_{2}\right\}_{+} & =\sum_{k=1}^{n}\left(\frac{\partial f_{1}}{\partial q_{k}} \frac{\partial f_{2}}{\partial p_{k}}+\frac{\partial f_{1}}{\partial p_{k}} \frac{\partial f_{2}}{\partial q_{k}}\right), \\
\left\langle f_{1} \mid f_{2}\right\rangle & =4 \sum_{k=1}^{n} \frac{\partial f_{1}}{\partial z_{k}} \frac{\partial f_{2}}{\partial \bar{z}_{k}} .
\end{aligned}
$$

3.2. Представление алгебры $\mathcal{O}$. Теперь рассмотрим соответствующее представление алгебры наблюдаемых. Прежде всего, справедлива следующая простая лемма.

ЛЕмма 5. Каждъй комплексный линейный оператор $A \in \mathfrak{g l}(n, \mathbb{C})$ определяет квадратичную функцию $f_{A}(\psi)=\frac{1}{2}\langle\psi \mid A \psi\rangle$. Функция вещественна, если и только если $A$ является эрмитовым, $A=A^{\dagger}$.

Введя соответствующие геометрические структуры, связанные с кэлеровой структурой, мы можем приступить к установлению связи между описанием на пространстве наблюдаемых $\mathfrak{u}(\mathcal{H})$ и описанием на гильбертовом пространстве $\mathcal{H} \equiv \mathbb{C}^{n}$.

ПРЕДЛОЖЕНИЕ 5. Отображение импульсов

$$
\mu: \mathcal{H} \rightarrow \mathfrak{u}^{*}(\mathcal{H}), \quad \mu:|\psi\rangle \mapsto \rho_{\psi}=|\psi\rangle\langle\psi|
$$

имеет следующие основные свойства:

- $\mu^{*}(\hat{A})=f_{A}$

- $\mu^{*}(\{\hat{A}, \widehat{B}\})=\left\{f_{A}, f_{B}\right\}$

- $\mu^{*}(\mathcal{R}(d \hat{A}, d \widehat{B}))=G\left(\mu^{*}(d \hat{A}), \mu^{*}(d \widehat{B})\right)$.

ДокАЗАТЕЛЬСТВо можно получить прямой проверкой.

Таким образом, можно заключить, что геометризация наблюдаемых на гильбертовом пространстве отвечает изучению соответствующих квадратичных функций.

3.3. Динамика. Теперь можно выразить динамику в гильбертовом пространстве (уравнение Шредингера), записав $i \hbar \frac{d}{d t}|\psi\rangle=H|\psi\rangle$ или, что эквивалентно, $\hbar \frac{d}{d t} f_{A}=\left\{f_{H}, f_{A}\right\}$. Последнее уравнение $\mu$-связано с уравнением Гейзенберга на пространстве наблюдаемых.

3.4. Собственные значения и собственные состояния. Далее при описании "геометризованного" гильбертова пространства нам нужно воспроизвести описание "собственных векторов" и “собственных значений”. Для этой цели разумно ввести ожидаемые значения, связанные с эрмитовыми операторами:

$$
e_{A}(\psi)=\frac{\langle\psi \mid A \psi\rangle}{\langle\psi \mid \psi\rangle} .
$$

Справедливы следующие утверждения.

1. Критические точки дифференциала $d e_{A}$ соответствуют собственным векторам оператора $A$.

2. Значения $e_{A}$ в критических точках являются соответствующими собственными значениями оператора $A$. 
ЗАмечаниЕ 2. Критические точки дифференциала $d e_{A}$ совпадают с критическими точками соответствующего гамильтонова векторного поля $\Omega\left(d e_{A}\right)$ или соответствующего градиентного векторного поля $G\left(d e_{A}\right)$.

ЗАмЕчаниЕ 3. Имеет место равенство

$$
G\left(d e_{A}, d e_{A}\right)=\frac{\left\langle\psi \mid A^{2} \psi\right\rangle}{\langle\psi \mid \psi\rangle}-\frac{\langle\psi \mid A \psi\rangle}{\langle\psi \mid \psi\rangle} \frac{\langle\psi \mid A \psi\rangle}{\langle\psi \mid \psi\rangle},
$$

т.е. $G\left(d e_{A}, d e_{A}\right)$ является дисперсией среднего значения наблюдаемой, соответствующей оператору $A$ в состоянии $|\psi\rangle$, а потому квадрат гамильтонова векторного поля, связанного с $A$, строго связан с "неопределенностью" в измерении $A$ в состоянии $|\psi\rangle$.

ЗАмЕчАниЕ 4. ГНС-конструкция, связанная с чистым состоянием, должна обеспечивать нас комплексным гильбертовым пространством размерности $n$, если мы исходим из эрмитовых $(n \times n)$-матриц. Поэтому ее можно рассматривать как способ построения "симплектической реализации" алгебраических структур на $\mathfrak{u}^{*}(\mathcal{H})$ (а также реализации алгебры Йордана).

\section{4. ЗАКЛЮЧЕНИЕ}

В настоящей работе предложен способ геометризации квантовой механики, основанный на множестве наблюдаемых квантовой системы. Для замыкания круга можно попробовать связать полученные нами результаты с обычными геометрическими конструкциями, основанными на пространстве состояний.

Вероятностная интерпретация квантовой механики требует, чтобы состояния были нормированы и, более того, поскольку нормированные векторы отождествляются с амплитудами вероятности, чтобы физически подходящие плотности вероятности были инвариантны относительно умножения векторов на фазовый множитель. Подводя итог, скажем, что физические состояния должны отождествляться не с векторами в гильбертовом пространстве, а скорее с точками в ассоциированном комплексном проективном пространстве. Таким образом, мы определяем главное слоение $\mathbb{C}_{0} \rightarrow \mathcal{H}-\{0\} \rightarrow \mathbb{P H}$, где $\mathbb{C}_{0}=\mathbb{C}-\{0\}-$ множество ненулевых комплексных чисел, а $\mathcal{H}-\{0\}$ - множество ненулевых векторов; $\mathbb{P} \mathcal{H}$ обозначает комплексное проективное пространство, ассоциированное с $\mathcal{H}$.

$\mathrm{C}$ точки зрения многообразий фактор $\mathbb{P} \mathcal{H}$ полностью описывается инволютивным распределением, порожденным инфинитезимальным генератором растяжений $\Delta$ и инфинитезимальным генератором умножения на фазу $J(\Delta)$. Они дают достаточно информации для описания точек проективного пространства. Однако тензоры $G$ и $\Omega$, введенные в предыдущем разделе, не проектируются в соответствии с указанным слоением. Получить из них проектируемые тензорные поля можно путем умножения на конформный множитель $\langle\psi \mid \psi\rangle$. Однако величина $\langle\psi \mid \psi\rangle \Omega$ не определяет скобку, удовлетворяющую тождеству Якоби. На самом деле, она порождает скобку Якоби. Поэтому скобку Пуассона, которую мы получаем в комплексном проективном пространстве, следует рассматривать как редукцию скобки Якоби, а не скобки Пуассона на $\mathcal{H}$. Подробности можно найти в работе [15]. 
Отметим, что комплексное проективное пространство можно отождествить с минимальными симплектическими орбитами на $\mathfrak{u}^{*}(\mathcal{H})$ [19]. Уравнения движения Шредингера в гильбертовом пространстве проектируются на уравнения движения фон Неймана в пространстве чистых состояний (проекторы имеют ранг единица), см. работу [23].

Этим наблюдением мы завершаем нашу работу, заметив, что (все) различные формализмы для описания квантовых систем можно “геометризовать", причем эти "геометризации" основаны на рассмотрении отображения импульсов, связанного с сильно гамильтоновым действием унитарной группы на гильбертовом пространстве состояний. Рассуждение было проведено для конечноуровневых квантовых систем. Однако расширение на бесконечномерные гильбертовы пространства сталкивается лишь с проблемами, которые можно назвать техническими, а концептуальные геометрические рамки полностью очерчены конечномерной ситуацией.

\section{Список литературы}

[1] G. Marmo, G. Morandi, "Some geometry and topology", Low Dimensional Quantum Field Theory for Condensed Matter Physicists, Lect. Notes of ICTP, eds. S. Lundqvist, G. Morandi, Yu Lu, World Sci. Publ., Singapore, 1995, 1-108.

[2] П. А. М. Дирак, Приниипы квантовой механики, Наука, М., 1979.

[3] E. Segal, Ann. Math. (2), 48 (1947), 930-948.

[4] R. Haag, D. Kastler, J. Math. Phys., 5 (1964), 848-861.

[5] R. Cirelli, P. Lanzavecchia, A. Manià, J. Phys. A, 16 (1983), 3829-3835.

[6] R. Cirelli, P. Lanzavecchia, Nuovo Cimento B, 79 (1984), 271-283.

[7] M. C. Abbati, R. Cirelli, P. Lanzavecchia, A. Manià, Nuovo Cimento B, 83 (1984), 43-60.

[8] A. Bloch, Trans. Amer. Math. Soc., 302 (1987), 787-796.

[9] A. Heslot, Phys. Rev. D, 31 (1985), 1341-1348.

[10] D. J. Rowe, A. Ryman, G. Rosensteel, Phys. Rev. A, 22 (1980), 2362-2373.

[11] T. R. Field, J. S. Anandan, J. Geom. Phys., 50 (2004), 56-78.

[12] D. Brody, L. P. Hughston, J. Geom. Phys., 38 (2001), 19-53.

[13] F. Strocchi, Rev. Mod. Phys., 38 (1966), 36-40.

[14] A. Ashtekar, T.A. Shilling, "Geometrical formulation of quantum mechanics", On Einstein's Path, Essays in honor of Engelbert Schucking (New York, 1996), ed. A. Harvey, Springer, New York, 1999, 23-65.

[15] V. I. Man'ko, G. Marmo, E. C. G. Sudarshan, F. Zaccaria, Rep. Math. Phys., 55 (2005), 405-422.

[16] P. Jordan, J. von Neumann, E. P. Wigner, Ann. Math. (2), 35 (1934), 29-64.

[17] G. G. Emch, Mathematical and Conceptual Foundations of 20th-Century Physics, NorthHolland Math. Stud., 100, North Holland, Amsterdam-New York-Oxford, 1984.

[18] N. P. Landsman, Mathematical Topics Between Classical and Quantum Mechanics, Springer Monogr. Math., 19, Springer, New York, 1998.

[19] J. Grabowski, M. Kuś, G. Marmo, J. Phys. A, 38 (2005), 10217-10244.

[20] J. Grabowski, M. Kuś, G. Marmo, Open Syst. Inf. Dyn., 13 (2006), 343-362.

[21] V. I. Man'ko, G. Marmo, P. Vitale, F. Zaccaria, Int. J. Mod. Phys. A, 9 (1994), 5541-5561.

[22] L. J. Boya, J. F. Cariñena, J. M. Gracia-Bondía, Phys. Lett. A, 161 (1991), 30-34.

[23] V. I. Man'ko, G. Marmo, E. C. G. Sudarshan, F. Zaccaria, J. Russ. Laser Res., 20 (1999), 421-437. 